\title{
BMJ Does comorbidity increase the risk Open of mortality among children under 3 years of age?
}

\author{
Christa L Fischer Walker, ${ }^{1}$ Jamie Perin, ${ }^{1}$ Jodi L Liu, ${ }^{1}$ Joanne Katz, ${ }^{1}$ \\ James M Tielsch, ${ }^{1,2}$ Robert Black ${ }^{1}$
}

To cite: Fischer Walker CL, Perin J, Liu JL, et al. Does comorbidity increase the risk of mortality among children under 3 years of age?. BMJ Open 2013;3:e003457. doi:10.1136/bmjopen-2013003457

- Prepublication history for this paper is available online. To view these files please visit the journal online (http://dx.doi.org/10.1136/ bmjopen-2013-003457).

Received 21 June 2013 Revised 29 July 2013 Accepted 30 July 2013

${ }^{1}$ Department of International Health, Johns Hopkins Bloomberg School of Public Health, Baltimore, Maryland, USA

${ }^{2}$ Department of Global Health, George Washington University, School of Public Health and Health Services, Washington, DCUSA

Correspondence to Dr Christa L Fischer Walker; cfischer@jhsph.edu

\section{ABSTRACT}

Objectives: Diarrhoea and pneumonia remain leading causes of morbidity and mortality in children under 5 years of age. Little data is available to quantify the burden of comorbidity and the relationship between comorbid diarrhoea and pneumonia infections and mortality. We sought to quantify the relationship between comorbidity and risk of mortality among young children in two community-based studies conducted among South Asian children.

Design: Secondary data analysis of two cohort studies.

Participants: We identified two cohort studies of children under 3 years of age with prospective morbidity at least once every 2 weeks and ongoing mortality surveillance.

Outcome measures: We calculated the mortality risk for diarrhoea and acute lower respiratory infection (ALRI) episodes and further quantified the risk of mortality when both diseases occur at the same time using a semiparametric additive model.

Results: Among Nepali children, the estimated additional risk of mortality for comorbid diarrhoea and ALRI was $0.0014(-0.0033,0.0060)$. Among South Indian children, the estimated additional risk of mortality for comorbid diarrhoea and ALRI was 0.0032 $(-0.0098,0.0162)$. This risk is in addition to the single infection risk of mortality observed among these children.

Conclusions: We observed an additional risk of mortality in children who experienced simultaneous diarrhoea and $\mathrm{ALRI}$ episodes though the $\mathrm{Cl}$ was wide indicating low statistical support. Additional studies with adequate power to detect the increased risk of comorbidity on mortality are needed to improve confidence around the effect size estimate.

\section{BACKGROUND}

Diarrhoea and pneumonia remain leading causes of morbidity and mortality in children under 5 years of age. ${ }^{1}$ Young children experience on average 2.9 episodes of diarrhoea per year with the highest rates among those between 6 and 18 months of age. ${ }^{2}$ Children under 2 years of age experience multiple

\section{ARTICLE SUMMARY}

\section{Article focus}

- Diarrhoea and pneumonia are leading causes of morbidity and mortality.

- Diarrhoea has been shown to be a risk factor for pneumonia.

- However, little is known about the role of comorbidity (ie, simultaneous infections) on risk of mortality.

Key messages

- We used data from two previously published cohort studies with routine morbidity surveillance and ongoing mortality monitoring to quantify the risk of mortality for a single illness and the added risk if coinfection occurred.

- There was no statistically significant additional risk of mortality for children with coinfections above and beyond the mortality risk of individual infection.

Strengths and limitations of this study

- Included data from two large studies conducted among South Asian children to answer this important question.

- Despite analytic rigour, sample sizes were too small to detect a statistically significant risk beyond the mortality risk of individual infection.

respiratory track infections per year and each year one in five will have a case of confirmed pneumonia. ${ }^{1}$ There is little data available to quantify the burden of comorbidity (ie, simultaneous infections) ${ }^{3-5}$ and even less data available to describe the relationship between comorbid infections as a risk factor for death. Thus far, there has been only one analysis of a community-based study to assess the risk of comorbidity on subsequent mortality. ${ }^{3}$ Although no significant association was found between comorbidity and risk of mortality in this study, an increased prevalence of comorbidity was observed with increased disease severity. We sought to further investigate the relationship between comorbidity and risk of mortality among 
young children in two additional community-based studies conducted among South Asia children.

\section{METHODS}

\section{Selection of data sets}

We conducted a literature review to identify communitybased studies that included prospective morbidity and mortality surveillance of children under 5 years of age in low-income or middle-income countries. We searched PubMed, Scopus and Google Scholar for studies published between 1980 and 2010. The following key words were used in the search: diarrh(o)ea, pneumonia, acute lower respiratory infection (ALRI), morbidity and mortality. Inclusion and exclusion criteria were established a priori. We sought studies with at least 12 months of morbidity and mortality surveillance. We included only those studies that had household morbidity surveillance at least once every 2 weeks to record daily diarrhoea and respiratory tract symptoms in the two previous weeks (or less) and those that included prospective mortality monitoring and used a standard verbal autopsy to determine cause of death. ${ }^{6}$ We also conducted an active search of past large community-based studies within the Child Health Epidemiology Reference Group (CHERG) network of investigators. Emails were sent to all possible investigators identified by CHERG members as those who might have a qualifying study.

\section{Methods of selected studies}

With this two-part process, we identified two studies that met all inclusion criteria. Both studies enrolled children under 3 years of age in South Asia. The detailed methods of both studies have been previously published. ${ }^{78}$ In the Nepal Nutrition Intervention ProjectSarlahi 4 (NNIPS-4), children 1-35 months of age living in the study areas were enrolled in a micronutrient supplementation trial and received daily zinc, iron, zinc and iron or placebo. Active morbidity surveillance was conducted twice a week in a subgroup of enrolled children who were followed for 1 year. In South India, investigators of the Vitamin A Supplementation in Newborns Study (VASIN) randomised infants at birth and followed them every 2 weeks, collecting daily accounts of infant morbidities, up to 6 months of age. In both studies, infant and child deaths were assessed using a standard verbal autopsy.
Study arm inclusion and case definitions

We included the iron and placebo arms from the NNIPS-4 study. Children receiving zinc were excluded because the zinc supplementation had a direct impact on mortality. ${ }^{7}$ From VASIN, we included both vitamin A and placebo arms of the study. While vitamin A supplemented children did have lower mortality rates, supplementation is now routine in parts of the world with vitamin A deficiency; thus, combining the two groups likely represents a more typical child population with variable vitamin A status and supplementation coverage.

We used prospective morbidity data to quantify the rates of diarrhoea and ALRI. Case definitions for diarrhoea and ALRI episodes were determined by the original studies (table 1). The definitions were identical for diarrhoea and varied slightly based on collected signs and symptoms for ALRI. In cases in which morbidity data were missing in the 2 weeks prior to death, we used data available from the retrospective questionnaire as part of the detailed verbal autopsies conducted on all child deaths. Questions from the retrospective data most resembling those asked in the morbidity data collection were used to supplement the prospective data. For both studies, it was assumed that deaths because of diarrhoea (definition in table 1) also included a diarrhoea episode in the 2 weeks prior to death. For VASIN, deaths because of fever, difficulty breathing and persistent cough in the verbal autopsy (VA) retrospective questionnaire were classified with ALRI morbidity in the 2 weeks prior to death. For NNIPS-4, deaths that indicated difficulty breathing and fever prior to death were classified with ALRI morbidity in the 2 weeks prior to death.

\section{STATISTICAL METHODS}

We sought to calculate the mortality risk for diarrhoea and ALRI episodes and further quantify the risk of mortality when both diseases occur at the same time. We used a semiparametric additive model to compare the mortality risk by episode type in the previous week while allowing estimated risk for those with no ALRI or diarrhoea to vary by age (our chosen time scale). ${ }^{9}{ }^{10}$ This regression model, defined by Aalen, allows for flexibility in the overall mortality rate, which is modelled non-parametrically for a given time scale, while the difference between mortality rates is assumed to be linear. Where model assumptions hold, the difference in mortality risk depending on

\section{Table 1 Case definitions for diarrhoea and ALRI used to define an episode of diarrhoea and/or ALRI}

\begin{tabular}{lll}
\hline & NNIPS-4 & VASIN \\
\hline Diarrhoea & 4 or more loose/watery stools per day with episodes & 4 or more loose/watery stools per day with episodes \\
& separated by at least 3 symptom free days & separated by at least 3 symptom free days \\
ALRI & Fever, cough or difficulty breathing with all 3 & Cough and difficulty breathing with fever on at least \\
& symptoms on at least 1 day during the episode with & 1 day during the episode with episodes separated by at \\
& 7 or more days between episodes & least 3 symptom free days \\
\hline
\end{tabular}

$\mathrm{ALRI}$,acute lower respiratory infection; NNIPS-4, Nepal Nutrition Intervention Project-Sarlahi-4; VASIN, Vitamin A Supplementation in Newborns Study. 
episode is quantified by a sum of group risks. We conducted the analysis first stratified by treatment groups within each study (ie, iron vs placebo for NNIPS-4 and vitamin A vs placebo for VASIN) and combined where no difference in the effect of comorbidity on mortality risk was observed between groups. All analyses were conducted with the open source software R 2.15.0 ${ }^{11}$ using the survival analysis package timereg. ${ }^{12}$

\section{RESULTS}

\section{Selected studies}

There were 2610 children enrolled in the NNIPS-4 morbidity substudy with a total of 1730 child-years of observation and 19 deaths (table 2). In VASIN, there were 11407 enrolled children who contributed to the active morbidity surveillance in the postnatal period (ie, from 28 days of age). Among these, there were 3720 child years of observation and 151 postneonatal deaths (table 2).

\section{MODEL RESULTS}

The results of the Aalen regression framework for both NNIPS-4 and VASIN are presented in table 3. We present the estimates for regression parameters and their approximate SE and CIs. These parameters are interpretable as the additional population mortality rate in 1 week, or the average additional risk of mortality within 1 week. The comorbidity parameter is for an interaction between indicators for ALRI and diarrhoea in the previous week. These risks are in addition to the base mortality rate, which is determined by age on a weekly basis for the duration of the study period (ie, for 12 calendar months of enrollment for NNIPS-4 and from 28 to 180 days for VASIN). Among Nepali children (NNIPS-4), the estimated additional risk of mortality for comorbid diarrhoea and ALRI was 0.0014 ( -0.0033 , $0.0060)$. For VASIN, we conducted all analyses stratified by supplement group and observed no interaction between intervention group and comorbidity risk and thus are presenting only the combined results here. Among South Indian children (VASIN), the estimated additional risk of mortality for comorbid diarrhoea and ALRI was $0.0032(-0.0098,0.0162)$.
The estimated risk of mortality from the Aalen regression model is shown for NNIPS-4 and VASIN in figures 1 and 2. We present the non-parametrically estimated mortality for those children with no ALRI or diarrhoea episode in the prior week. The estimated risk of mortality for those with an episode of ALRI alone, with diarrhoea alone and with both ALRI and diarrhoea in the prior week is determined by the sum of this nonparametric risk and the survival regression parameters for each group as shown in table 3.

\section{DISCUSSION}

We present the risk of mortality due to diarrhoea and ALRI episodes individually, and the additive risk as a result of a child having both diarrhoea and respiratory infections simultaneously. For these analyses, we combined the micronutrient intervention groups after first assessing each independently and observing no interaction. By combining the results, the data are more representative of an average low-income and middle-income population of young children with a wide range of nutritional states. The Cox proportional hazards model is often used for analysing cohort mortality data like what we included here from the NNIPS-4 and VASIN studies; however, the Cox model assumes that the ratio of mortality rates is constant over time. This can be a difficult assumption when examining the interaction of effects as in our case with comorbidity. The Cox model is also difficult to interpret, because mortality risk due to multiple effects is often assumed additive and Cox parameters are multiplicative. Cohort mortality data has also been analysed with generalised estimating equations (GEE). ${ }^{3}$ GEE is an improvement with regard to the flexibility for non-proportional mortality rates, but is sensitive to missing outcomes, which is a potential issue for cohort survival data. ${ }^{13}$ We chose to use an additive model $^{9}{ }^{10}$ that allows for more flexibility in comparing mortality rates over time, especially for covariates that vary within individuals, ${ }^{14}$ as in our case for recent ALRI and diarrhoea symptoms as risk factors for mortality. Our primary interest is also to specify the risk of mortality for children with comorbid symptoms as a sum of the risk due to ALRI and that due to diarrhoea. This analysis

Table 2 Mortality in the NNIPS-4 morbidity substudy and the VASIN study by intervention arm

\begin{tabular}{|c|c|c|c|c|c|c|}
\hline Study arm & $\begin{array}{l}\text { Number of children } \\
\text { observed }\end{array}$ & $\begin{array}{l}\text { Observation time } \\
\text { (years) }\end{array}$ & Deaths & $\begin{array}{l}\text { Mortality rate } \\
\text { (per year) }\end{array}$ & $\begin{array}{l}\text { ALRI } \\
\text { deaths* }\end{array}$ & $\begin{array}{l}\text { Diarrhoea } \\
\text { deaths }^{*}\end{array}$ \\
\hline \multicolumn{7}{|l|}{ NNIPS-4 } \\
\hline Iron & 576 & 366.2 & 4 & 0.0109 & 0 & 0 \\
\hline Placebo & 2034 & 1363.8 & 15 & 0.0110 & 2 & 5 \\
\hline \multicolumn{7}{|l|}{ VASIN } \\
\hline Placebo & 5713 & 1860.3 & 84 & 0.0452 & 19 & 18 \\
\hline Vitamin A & 5694 & 1859.9 & 67 & 0.0360 & 22 & 11 \\
\hline
\end{tabular}


Table 3 Additive model of mortality risk for Nepali (NNIPS-4) and South Indian (VASIN) children, Aalen additive hazards model with robust SEs, combining treatment groups

\begin{tabular}{|c|c|c|c|c|c|c|}
\hline & \multirow[b]{2}{*}{ Estimate } & \multirow[b]{2}{*}{ SE } & \multirow[b]{2}{*}{$\mathbf{Z}$} & \multirow[b]{2}{*}{$\mathbf{P}$} & \multicolumn{2}{|l|}{$95 \% \mathrm{Cl}$} \\
\hline & & & & & Lower & Upper \\
\hline \multicolumn{7}{|l|}{ Nepal } \\
\hline Diarrhoea, but no ALRI & 0.0002 & 0.0002 & 1.07 & 0.287 & -0.0002 & 0.0006 \\
\hline ALRI, but no diarrhoea & 0.0007 & 0.0005 & 1.33 & 0.185 & -0.0003 & 0.0016 \\
\hline $\begin{array}{l}\text { Additional risk associated with having both conditions } \\
\text { simultaneously }\end{array}$ & 0.0014 & 0.0024 & 0.573 & 0.567 & -0.0033 & 0.0060 \\
\hline \multicolumn{7}{|l|}{ South India } \\
\hline Diarrhoea, but no ALRI & 0.0045 & 0.0008 & 5.55 & $<0.001$ & 0.0029 & 0.0061 \\
\hline ALRI, but no diarrhoea & 0.0034 & 0.0011 & 2.98 & 0.003 & 0.0012 & 0.0056 \\
\hline $\begin{array}{l}\text { Additional risk associated with having both conditions } \\
\text { simultaneously }\end{array}$ & 0.0032 & 0.0066 & 0.49 & 0.628 & -0.0098 & 0.0162 \\
\hline
\end{tabular}

allows us to test whether mortality risk is truly additive over time, and if so, to compare the sum of risks and to make inference on the difference between sums of risks.

We did observe an additional risk of mortality among children who experienced simultaneous diarrhoea and ALRI episodes in both the NNIPS-4 and VASIN data sets, but both estimates had wide CIs and thus we lacked statistical power. There are several possible features in the data that may have contributed to these results. First, the actual synergism between ALRI and diarrhoea may be very close to zero (or less than zero), and the predicted positive synergism a randomly observed phenomena. It is also possible that lack of data, particularly low observed mortality rates on average for each cohort, together with the small number of individuals with comorbidity symptom classification, may have made a meaningful comorbid effect difficult to detect. The low mortality rates may be the result of a study Hawthorne

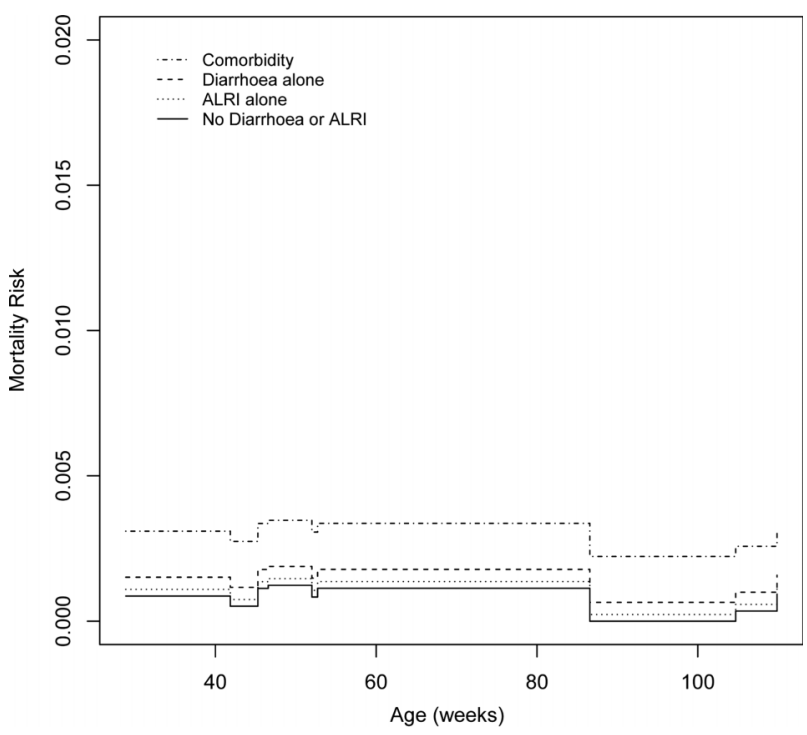

Figure 1 Mortality rates estimated by the linear survival regression model in the Nepal data set, conditional on episode type in the previous week and age in weeks. effect. In both studies, morbidity surveillance was routine; sometimes involvement in a study and the awareness that someone is coming to ask questions about the health of the child can increase care-seeking behaviour such that children enrolled in the study would be more likely to receive timely care than those who were not in the study. Prompt and appropriate care seeking for diarrhoea and pneumonia will decrease diseases severity and overall mortality from these two diseases. The total number of deaths in the NNIPS-4 study was particularly low, but even with 151 postnatal deaths in VASIN, only three of these deaths were associated with comorbidity between ALRI and diarrhoea symptoms.

At the start of our search for data sets fitting our tight inclusion and exclusion criteria, we were hopeful that rigorous searching would uncover more than two studies. Although there are many large-scale studies

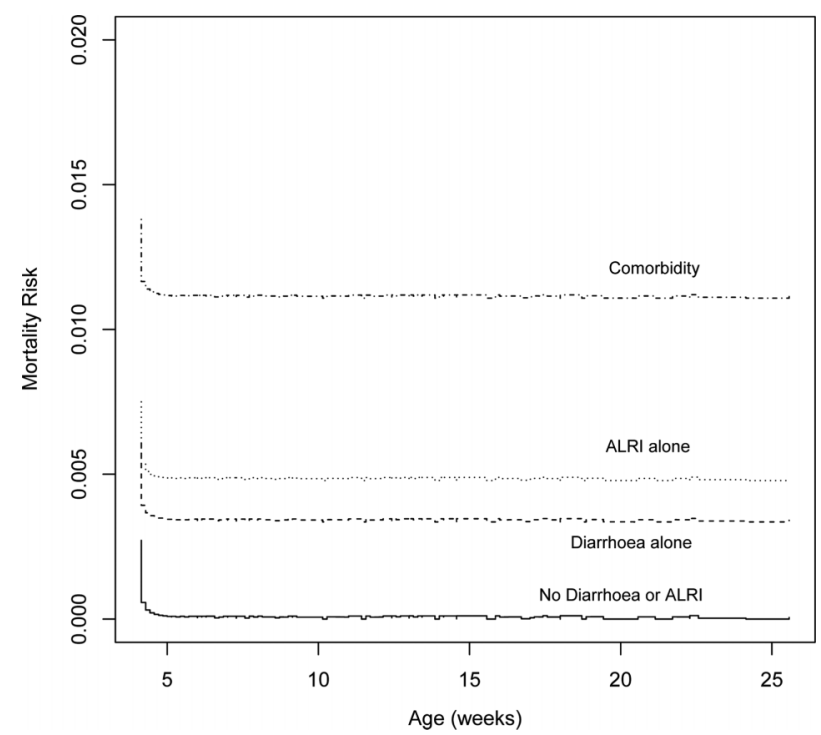

Figure 2 Mortality rates estimated by the linear survival regression model in the South India data set, conditional on episode type in the previous week and age in weeks. 
monitoring mortality among young children and many other studies with active morbidity surveillance, combining these two attributes limited the studies available for inclusion. Because of the demands of routine household visits, sample sizes are rarely large enough to include mortality and all such studies would require community involvement and large budgets. Given the intense nature of conducting a study with active morbidity surveillance and the large sample size needed to complete a study with verbal autopsies, it is unlikely that we missed eligible studies through our intense search process.

There are several pathways that may explain the added risk of mortality observed among children with simultaneous diarrhoea and ALRI infections. Several studies have reported diarrhoea as a risk factor for ALRI ${ }^{15-17}$; these children may have begun with an episode of diarrhoea which leads to nutritional setbacks, compromising the immune system, and thus leaving the child susceptible to subsequent infection ${ }^{18}$ and the development of more severe infections increasing the risk for mortality. ${ }^{19}$ It is also possible that respiratory infection or the immune response to it may alter the gut flora increasing susceptibility to or severity of intestinal infections. ${ }^{20}$ It is difficult in community-based studies to determine the exact timing of infection and illness because presentation with signs or symptoms may be several days after initial infection. Furthermore, in communities in developing countries, children have high rates of infection due to poor environmental conditions and even simultaneous infectious illnesses occurring by chance could result in additive or greater risk of death. Several studies have reported an increased risk of mortality among children hospitalised with multiple infections (including respiratory signs and symptoms) ${ }^{21}$ and the presence of multiple conditions reported among children who died in hospital settings despite cause of death being assigned to one disease. ${ }^{22}$ Although the concept of comorbidity as an increased risk may be noted in the literature and acknowledged clinically among pediatricians, we lack the evidence to accurately quantify the value of this added risk and most importantly to then understand what interventions could be put in place to prevent dual severe infections leading to mortality.

Further study could shed light on these issues and the underlying relationships between child mortality, ALRI and diarrhoea. Several issues would need to be considered to plan a study for detecting differences in mortality rates for comorbidity compared to the additive risk of ALRI and diarrhoea alone. Of primary concern is the study size. To estimate the size of a study that would be needed to observe a statistically significant effect, we conducted a power analysis assuming that the ratio of comorbidity to single disease episodes observed in the South Indian data would be observed in future studies, that is, that for every 100 children with ALRI or diarrhoea symptoms alone, 4 children would have both ALRI and diarrhoea symptoms simultaneously. We also assumed that among children ever reporting comorbidity, the weekly point prevalence of comorbid symptoms would be again consistent with the VASIN data (ie, 23\%) and that overall mortality rates would be similar to those in the VASIN data. Given these assumptions, and similar incidence rates to those in the VASIN data, a study enrolling children at 1 month and following them until 6 months of age would need to enroll 35900 infants to have $80 \%$ power to find a significant comorbid effect on the mortality rate with a type 1 error rate of $5 \% .^{23}$ With similar incidence rates to those observed in VASIN, a study of this size would produce 8720 children with ALRI or diarrhoea symptoms during the study period, a critical number determining power in a study of the comorbidity effect on mortality.

A study of this magnitude is not likely in the near future and may be even harder to envision as under five mortality rates decline in many parts of the world. Despite this, diarrhoea and pneumonia remaining the leading causes of death among children under 5 years of age in low-income and middle-income countries ${ }^{1}$ and the possibility that joint infection could increase the risk for mortality is important to recognise. This knowledge highlights the importance of prevention of both diseases with improved water and sanitation and nutrition, and appropriate case management.

Acknowledgements The authors thank the children and families who participated in the studies and the original study teams who were part of data collection for these important trials.

Contributors CLFW and REB conceptualised the study and led the search for data sets. JP and JLL conducted the analysis. JK and JMT were primary investigators on the original studies and contributed extensively to the analysis and broader understanding of the data. CLFW and JP wrote the first draft of the paper. All authors contributed to the final draft and approved the final version of the manuscript.

Funding The work was supported by a grant to the Child Health Epidemiology Reference Group (CHERG) from the Bill and Melinda Gates Foundation via the US Fund for UNICEF.

Competing interests None.

Provenance and peer review Not commissioned; externally peer reviewed.

Data sharing statement No additional data are available.

Open Access This is an Open Access article distributed in accordance with the Creative Commons Attribution Non Commercial (CC BY-NC 3.0) license, which permits others to distribute, remix, adapt, build upon this work noncommercially, and license their derivative works on different terms, provided the original work is properly cited and the use is non-commercial. See: http:// creativecommons.org/licenses/by-nc/3.0/

\section{REFERENCES}

1. Walker CL, Rudan I, Liu L, et al. Global burden of childhood pneumonia and diarrhoea. Lancet 2013;381:1405-16.

2. Fischer Walker CL, Perin J, Aryee MJ, et al. Diarrhea incidence in low- and middle-income countries in 1990 and 2010: a systematic review. BMC Public Health 2012;12:220.

3. Fenn B, Morris SS, Black RE. Comorbidity in childhood in northern Ghana: magnitude, associated factors, and impact on mortality. Int $J$ Epidemiol 2005;34:368-75.

4. Fischer Walker CL, Perin J, Katz J, et al. Diarrhea as a risk factor for acute lower respiratory tract infections among young children in low income settings. J Global Health 2013;3:1-8.

5. Schmidt WP, Cairncross S, Barreto ML, et al. Recent diarrhoeal illness and risk of lower respiratory infections in children under the age of 5 years. Int J Epidemiol 2009;38:766-72.

6. Anker M, Black RE, Coldham $\mathrm{C}$, et al. A standard verbal autopsy method for investigating causes of death in infants and children. Geneva: World Health Organization, 1999.

7. Tielsch JM, Khatry SK, Stoltzfus RJ, et al. Effect of daily zinc supplementation on child mortality in southern Nepal: a 
community-based, cluster randomised, placebo-controlled trial. Lancet 2007;370:1230-9.

8. Tielsch JM, Rahmathullah L, Thulasiraj RD, et al. Newborn vitamin A dosing reduces the case fatality but not incidence of common childhood morbidities in South India. J Nutr 2007;137:2470-4.

9. Aalen OO. A linear regression model for the analysis of life times. Stat Med 1989;8:907-25.

10. Lin DY, Ying Z. Semiparametric analysis of the additive risk model. Biometrika 1994;81:61-71.

11. R: A Language and Environment for Statistical Computing [program]. Vienna, Austria: R Foundation for Statistical Computing, 2013.

12. T.M. Scheike. Dynamic regression models for survival data. New York, NY: Springer, 2006.

13. Zeger SL, Liang KY. Longitudinal data analysis for discrete and continuous outcomes. Biometrics 1986;42:121-30.

14. Aalen $\mathrm{OO}$. Further results on the non-parametric linear regression model in survival analysis. Stat Med 1993;12:1569-88.

15. Walker CL, Perin J, Katz J, et al. Diarrhea as a risk factor for acute lower respiratory tract infections among young children in low income settings. J Global Health 2013;3:10402.

16. Coles CL, Fraser D, Givon-Lavi N, et al. Nutritional status and diarrheal illness as independent risk factors for alveolar pneumonia. Am J Epidemiol 2005;162:999-1007.
17. Schmidt WP, Cairncross S, Barreto ML, et al. Recent diarrhoeal illness and risk of lower respiratory infections in children under the age of 5 years. Int J Epidemiol 2009;38:766-72.

18. Guerrant RL, Oria RB, Moore SR, et al. Malnutrition as an enteric infectious disease with long-term effects on child development. Nutr Rev 2008;66:487-505.

19. Black RE, Victora CG, Walker SP, et al. Maternal and child undernutrition and overweight in low-income and middle-income countries. Lancet 2013;382:427-51.

20. Stecher B, Hardt WD. The role of microbiota in infectious disease. Trends Microbiol 2008;16:107-14.

21. Islam SS, Khan MU. Risk factors for diarrhoeal deaths: a case-control study at a diarrhoeal disease hospital in Bangladesh. Int J Epidemiol 1986;15:116-21.

22. Mitra AK, Khan MR, Alam AN. Complications and outcome of disease in patients admitted to the intensive care unit of a diarrhoeal diseases hospital in Bangladesh. Trans $R$ Soc Trop Med Hyg 1991;85:685-7.

23. Lachin JM, Foulkes MA. Evaluation of sample size and power for analyses of survival with allowance for nonuniform patient entry, losses to follow-up, noncompliance, and stratification. Biometrics 1986;42:507-19. 